\title{
Role of the cancer registries in determining cancer mortality in Asia
}

\author{
Balkrishna B.Yeole \\ Mumbai Cancer Registry, Indian Cancer Society, Mumbai, India
}

\begin{abstract}
Background. In the absence of dependable data from the Civil Registration System (CRS) many countries have developed their own Sample Registration System (SRS). Under this scheme in India the SRS collects the information on fertility and mortality indicators at state and national levels.

Conclusions. In Asia in general and in India in particular cancer registries have played a crucial role in providing the improved cancer mortality data.
\end{abstract}

Key words: cancer registry; cancer mortality; sample registration system

\section{Introduction}

The burden of disease can be assessed through a number of epidemiological parameters such as incidence, prevalence, mortality and disability caused by the disease. The global burden of disease study conducted by WHO, World Bank and Harvard School of Public in 1990s, further developed the sophisticated epidemiological parameters on mortality, morbidity and disability to provide a composite index on the burden of disease like YLD (Years of Life Lived with Disability) and DALY (composite Index of Burden of Disease). However, data on incidence, prevalence,

Received 16 October 2007

Accepted 30 October 2007

Correspondence to: Dr. Balkrishna B.Yeole, M.Sc., Ph.D., Director, Mumbai Cancer Registry, Indian Cancer Society, 74, Jerbai Wadia Road, Parel, Mumbai400 012, India; Phone: +91-22-24122351; Fax: +91-2224122351; E-mail: bcrics@vsnl.com

Paper was presented at the $27^{\text {th }}$ Conference of International Association of Cancer Registries, Ljubljana, Slovenia, 17-20 September 2007. and disease specific mortality are frequently incomplete, not very reliable or are lacking in many countries particularly in Asia and Africa.

In the absence of dependable data from the Civil Registration System (CRS) many countries have developed their own Sample Registration System (SRS). Under this scheme in India the SRS collects the information on fertility and mortality indicators at state and national levels. The SRS mechanism involves collections of data through two different procedures. It is performed by continuous enumeration and retrospective half yearly survey by a process of matching two records and subsequent field verification of unmatched and partially matched events. The methodology provides a cross check on the correctness of events of births and deaths listed in both records. ${ }^{1}$

In order to estimate the cancer mortality from other than cancer registries there is a paucity of adequate data on the one hand and the complex pathogenesis of disease 
on the other one which make for complexity, particularly in rural population. In India, major death registration sources are neither reliable nor complete. A large percentage of cases goes unregistered and out of registered cases only, $10 \%$ of deaths are medically certified. ${ }^{2}$

In this contest it is important to mention that quality of death reporting to the system in less developed countries including India is very poor. Due to several socio-economic constraints the cause is not adequately noted in the death certificates. A sample registration system practice in India helps in this but for correlating with cancer registry data this is not optimally helpful. When cancer morbidity figures from SRS system and cancer registry are compared the SRS figures are at low levels. ${ }^{2}$

\section{The procedure adopted by cancer registries in determining cancer mortality}

There are three main sources of cancer deaths to be collected in the registry: Vital statistics departments of Municipal Corporation, Medical records department of collaborative hospitals and Active follow-up through telephone, postal enquiries and house visits.

In cancer registries, completeness of registrations, certification of deaths, disease coding practices, cause of death, basic information like address and demographic information and especially the duration of stay and primary cause of death are the main problems to be encountered.

There are number of reasons for the under registration of cancer deaths in cancer registries. The death certificates are not available at source of information. The death might have occurred out side the area of registration. Deaths may not have been registered at the vital statistics department of Municipal Corporation. If the cancer has long survival and death was not due to cancer, there is a high probability that the information that the disease had cancer had been totally forgotten. The migration also plays an important role. The death registration system may be defective at the vital statistics department. The cause of deaths may be erroneously reported like old age, etc.

Mumbai Cancer Registry was established in 1963 for Mumbai Municipal Corporation area. At present this registry covers about 12 million population having $437.7 \mathrm{sq} \mathrm{km}$ area. The death registration system in Mumbai is quite complete i.e. $98.7 \%{ }^{3}$ Reporting of cause of death as far as cancer is concerned is quite good - non site-specific deaths are less than 1\%. At present 9500 incidence cases are registered at this registry. From the department of Vital Statistics of Municipal Corporation 6200 cancer deaths are collected out of which 5000 are residents, 800 are non residents and 400 are not known residents. ${ }^{4}$

The method of collection of cancer death information in this registry is that the staff of the registry visits the Municipal Corporation's office to scrutinize all the deaths and copies information on deaths mentioning cancer of tumour which is the primary or secondary cause of death. Residents' deaths to incidence cases give $\mathrm{M} / \mathrm{I}$ Ratio. For Mumbai it is $52.6 \%$ which is quite comparable with European and developed countries.

The municipal Corporation also publishes the annual report on vital statistics. ${ }^{5}$ When the comparison is made for the year 2000, the information about the number of cancer deaths has two sources: the Municipal report has reported only 4320 cancer deaths, whereas the registry collected 6200 cancer deaths. This implies that the registry has recorded 1880 more cancer deaths $(30 \%)$ than the published report by the Municipal Corporation. 
Reasons for less cancer deaths reporting in the Municipal Corporation report may be due to the fact that they have been looked only at the primary cause of death and overlooked the secondary or underlying cause of death.

When the site specific deaths are compared in both reports, deaths due to secondary sites, glands, brain tumours, leukaemia, were very minimally reported in the corporation reports. This may be due to the lack of training of the coder of the vital statistics department. In short, all the deaths recorded at Vital statistics should be scrutinized by the trained registry staff.

In India, other than Mumbai registry, the method applied for few registries for the improving cancer mortality is described below.

It is well known that in India except Mumbai the death registration system is quite incomplete and the cause of death reporting system is not at satisfactory level. When the registry started functioning, the $\mathrm{M} / \mathrm{I}$ ratios were for Chennai in 1982 23\%, for Bangalore in 1982 17\%, for Bhopal in 1987 19\% and for Delhi in 1987 19\%.

The Chennai registry was established in 1982 by the cancer institute. At present, it covers the population of 4.3 million having an area of $170 \mathrm{sq} \mathrm{km}$. Having the incomplete reporting of cancer deaths and poor notification of cause of deaths this registry has improved the problem of under registration by the following way. This registry records all the deaths regardless of the cause of death from the vital statistics department and hospital records. Then, all the deaths are computerized. All this mortality data are matched with morbidity data. Matched deaths are then updated in morbidity data. Unmatched cancer deaths are then traced back by house visits. Cases with no other details are registered as "DCO's". By this method the M/I ratio of this registry has been improved from $24 \%$ to $54 \%{ }^{6}$
Indian Council of Medical Research, New Delhi has established the population based registry at Bhopal, in 1986 with the aim to evaluate the cancerogenic effect of Mythel Isocynate and cancer. This registry covers $285 \mathrm{sq} \mathrm{km}$ area having the population of 1.4 million. A death registration system is far from adequate resulting in under registration of the cancer mortality. This registry has identified burial grounds and crematoriums for the death registration system implementing the same methodology as of Chennai PBCR. It has been shown that M/ I ratio which was around 19\% initially has gone up to $36 \%$. $^{7}$

Tata Memorial Hospital, Mumbai, in collaboration with Indian Council of Medical Research, New Delhi, established the first rural cancer registry at Barshi in Solapur district of Maharashtra in 1987. At present it covers 0.4 million population with an area $3717 \mathrm{sq} \mathrm{km}$. Information on deaths is collected from the village death records and also from the local community. As death records are not generally medically certified, the relatives of all diseased are contacted to collect the relevant information to assist in "follow-track" to the medical records in the treating hospital or physician to identify the proven cancer cases. In this registry the $\mathrm{M} / \mathrm{I}$ ratio is $79 \%{ }^{8}$

Many registries collect the follow-up information for the survival studies. This procedure is also helpful to improve the cancer mortality in registries. Mumbai cancer registry collects the follow-up information for most of the major sites after 5 years for each case. To get the follow-up information, the methodology is used, firstly, to match with the cancer deaths collected from the vital statistics department (50\%). The follow-up information from the remaining patients are done by telephone and postal enquiries $(15 \%)$ and by house visits $(10 \%)$. Due to this procedure there have been improvements in cancer mortality about $10 \% .^{9}$ 
Special cross sectional surveys in registration areas are also helpful in improving cancer mortality statistics of the registry. Tata Institute of Fundamental Research, Mumbai has carried out a special health survey for Mumbai City population during 1991-94. In this survey the information on deaths has been also collected when survey data and Mumbai Registry data has been matched for the cancer mortality. It has been observed that there has been an improvement of $4.2 \%$ in cancer mortality.

It has been shown that there is an effective use of cancer registries for cancer survivorship research. ${ }^{10}$ Two hospital cancer registries in USA were used to recruit a large sample of breast cancer survivors for a study examining the late reproductive effects of breast cancer treatments. These two participative cancer registries were an excellent source of identifying a large sample for long term for breast cancer survivors. Although there are some limitations to this approach including a non response of a significant number of breast cancer survivors, tumour registries represent an important resource for the rapid identification of cancer survivors for research studies. Findings from this study suggest several enhancements for the future study that may increase the yield from registry recruitments.

Cancer mortality through cancer registries in Asia, Africa, may be improved as follows: it is well known that the usual method of mortality data collection as in the west will not give reliable and complete data. It is absolutely necessary that the improvements in the system of registration of deaths include the implementation of standard core information mortality form in all hospital-nursing homes in registration area and at birth and death registration units of the vital statistics department and at burial grounds and crematoriums. The improvements in the system of certification of the cause of death stress on the underly- ing and antecedent cause of death should be given. The medical personnel should be educated on the method of certifying the cause of death. The cancer registration topic should be introduced in curriculum of the final year MBBS at least one question on this topic in any clinical subject. Verbal autopsies have to be more rigorous and standardized procedures before the exact cause of death can be ascertained.

\section{Conclusions}

The cancer mortality assess is an important function of any cancer control programme. Cancer registries of the sound system are used for evaluating cancer mortality. Because of the scientific discipline in cancer registration system, the mortality rates obtained through cancer registry will be optimally productive. In Asia in general and in India in particular cancer registries have played a crucial role in providing the improved cancer mortality data.

\section{References}

1. Sample Registration System. Statistical Report 1998. New Delhi, India: Registrar General; 2000.

2. Ramanakumar AV, Yeole BB. Assessing cancer burden in rural India: An Analysis by cause of death statistics. Asian Pac J Cancer Prev 2005; 6: 221-3.

3. Gupta RB, Rao GR. Effect of elimination of different causes of death on expectation of life-Bombay, 1960-61. Indian J Med Res 1973; 61: 950-61.

4. Kurkure AP, Yeole BB, Sunny L, Koyande SS. Cancer incidence and mortality in Greater Mumbai, 2001. Mumbai, India: Indian Cancer Society: 2005.

5. Municipal Corporation of Greater Mumbai. Annual Report- 2001. Mumbai, India, 2003.

6. Shanta V, Swaminathan $\mathrm{R}$, Cancer incidence and mortality in Chennai, 2001. Chennai, India: Cancer Institute; 2004. 
7. Bharadwaj AK, Shrivastav A, Cancer incidence and mortality in Bhopal. Annual Report 2001. Bhopal, India; 2005.

8. Dinshaw KA, Nene BM. Cancer incidence and mortality in Barshi -2001. Annual Report. Barshi, India; 2005.

9. Sankarnarayan R, Black RJ, Parkin DM. Cancer survival in developing countries. IARC Scientific Publication No.145. Lyon, France: IARC. 1998.

10. Pakitil AT, Khan BA, Petersen L, Abraham LS, Greendale GA, Ganj PA. Making effective use of tumour registries in cancer survivorship. Cancer 2001; 92: 1305-14. 